\title{
Stratégie efficace et peu coûteuse pour la détection du cancer de la prostate à un stade précoce alors que la guérison est possible
}

\author{
Fernand Labrie
}

La combinaison, chez l'homme après 50 ans, d'un dosage systématique de l'antigène prostatique (PSA) et, en cas de concentration supérieure à $3 \mu \mathrm{g} / \mathrm{l}$, d'une échographie transrectale devrait permettre la détection précoce des cancers de la prostate d̀ un stade où un traitement chirurgical curateur est encore possible. Selon F. Labrie, la généralisation d'une telle attitude pourrait augmenter de dix ans l'espérance de vie des malades développant un cancer de la prostate, c'està-dire augmenter d'une année l'espérance de vie générale moyenne des hommes si un traitement efficace du cancer au stade précoce est appliqué.

TIRÉS A PART

F. Labrie.

$m / s n^{\circ} 7$, vol. 8 , septembre 92 e cancer de la prostate est maintenant le cancer le plus fréquent chez l'homme et il est la deuxième cause de décès dus au cancer [1]. De fait, aux États-Unis seulement, l'on prévoit que 132000 nouveaux cas de cancer de la prostate vont être mis en évidence en 1992, alors que 34000 hommes vont mourir de cette maladie pendant la même période. La probabilité de développer un cancer de la prostate menace 1 homme sur 11 au cours de sa vie, et cette incidence augmente continuellement. Le cancer de la prostate est habituellement diagnostiqué à un stade avancé

Malheureusement, avec les techniques diagnostiques actuellement utilisées, le cancer de la prostate est découvert, dans plus de la moitié des cas, à un stade avancé de la maladie alors qu'il a déjà migré à l'extérieur de la prostate et a atteint les os. A ce stade très avancé, le meilleur traitement disponible est le traitement combiné mis au point par le groupe de recherche du Centre hospitalier de l'université Laval [2]. Ce traitement - dont les résultats ont été confirmés par des études contrôlées, prospectives et à double insu réalisées par l'Institut du cancer des États-Unis [3] et l'EORTC (European organization on research and treatment of cancer) en
Europe [4] - est le premier et le seul à assurer, dans de bonnes conditions, la prolongation de la vie, mais la guérison ne peut malheureusement pas être offerte au patient [5].

Une des principales raisons du diagnostic tardif du cancer de la prostate est l'absence de douleurs ou autres symptômes au cours des nombreuses années pendant lesquelles la tumeur progresse au niveau de la prostate elle-même. Si l'on désire mettre en évidence le cancer de la prostate à un stade précoce, l'on ne doit pas compter sur une stratégie reposant sur l'apparition de symptômes ou de signes cliniques. En effet, lorsque les symptômes ou les signes cliniques deviennent perceptibles, il est habituellement trop tard pour offrir un traitement curatif.

Toutefois, il est bien reconnu qu'un diagnostic précoce, à un moment où le cancer est encore limité à la prostate, permettrait l'utilisation de techniques curatives qui ne sont pas disponibles à un stade plus avancé de la maladie, soit la prostatectomie radicale et, possiblement, la radiothérapie. Malgré le fait que le traitement précoce soit le seul espoir d'offrir la guérison du cancer de la prostate, relativement peu d'attention et d'efforts de recherche ont été dévoués au diagnostic et au traitement précoces de cette maladie. 
Une fenêtre de quelques années devrait permettre de mettre en évidence le cancer de la prostate avant qu'il ne migre à l'extérieur de la prostate

Les données de Stamey et al. [6] ont clairement démontré que le cancer de la prostate dont le volume est plus petit que $4 \mathrm{~cm}^{3}$ a une faible chance d'avoir migré à l'extérieur de la prostate et il est donc potentiellement guérissable, alors que les tumeurs de plus grand volume sont déjà très probablement accompagnées de métastases dans les os. Les techniques diagnostiques actuellement disponibles ne possèdent pas le niveau de sensibilité requis pour mettre en évidence le cancer de la prostate au cours des premières années qui suivent l'apparition de la première cellule cancéreuse. Toutefois, il existe une fenêtre de quelques années pendant lesquelles le cancer est encore localisé au niveau de la prostate, mais où le volume de la tumeur est suffisamment grand pour permettre sa détection par la technologie actuelle. Il est donc d'importance primordiale de mettre en évidence le cancer de la prostate à ce stade précoce avant qu'il ne pénètre la capsule prostatique et ne migre vers les ganglions lymphatiques et, à plus grande distance, dans les os.

Techniques disponibles pour le diagnostic précoce du cancer de la prostate

Trois techniques sont présentement disponibles pour la mise en évidence du cancer de la prostate à un stade précoce :

- Mesure du PSA sérique (prostatic specific antigen). Le PSA est une protéine sécrétée exclusivement par les cellules prostatiques. Toutefois, cette protéine est produite non seulement par les cellules prostatiques cancéreuses, mais également par le tissu normal. Comme la majorité des hommes, à partir de l'âge de 45 ans, peuvent souffrir d'une hypertrophie de la prostate (connue sous le nom d'hyperplasie bénigne de la prostate), une augmentation de la concentration de PSA dans la circulation peut être due à la sécrétion de cette protéine par les cellules cancéreuses, par les cellules hyperplasiques ou à la fois

plasiques. Ce problème de l'origine ambiguë d'une augmentation du PSA sérique a empêché l'utilisation de la mesure du PSA comme test fiable pour le dépistage du cancer de la prostate.

- Toucher rectal. Jusqu'à maintenant, la technique standard pour le diagnostic précoce du cancer de la prostate a été le toucher rectal. Toutefois, cette technique est relativement peu sensible, spécialement pour les tumeurs de petit volume. Lorsque le toucher rectal est utilisé seul pour la mise en évidence du cancer de la prostate, les tumeurs ainsi détectées ont fréquemment déjà atteint un volume trop grand et le cancer a déjà migré à l'extérieur de la prostate au moment du premier diagnostic.

- Échographie transrectale de la prostate. Les améliorations technologiques récentes de l'échographie permettent de mettre en évidence des tumeurs plus petites que celles détectables par le toucher rectal. En effet, le nombre de cancers mis en évidence par l'échographie transrectale de la prostate est environ le double de ceux diagnostiqués par le toucher rectal [7, 8]. Toutefois, cette technique est trop dispendieuse et requiert une participation trop lourde du personnel médical spécialisé pour être utilisée en routine de dépistage du cancer de la prostate dans la population en général.

Première étude de l'incidence du cancer de la prostate dans une population choisie au hasard

Bien que la mesure du PSA sérique, le toucher rectal et l'échographie transrectale de la prostate soient connus depuis plus de dix ans, aucune étude prospective n'avait été faite afin de déterminer les avantages relatifs de ces trois techniques utilisées chez une population d'hommes choisis au hasard et n'ayant pas de symptômes ou de signes particuliers de maladie prostatique. De plus, ce qui est d'importance majeure, aucune étude n'avait été faite afin de connaître l'incidence du cancer de la prostate dans la population en général grâce à l'examen de sujets choisis au hasard.

Comme partie d'une étude plus générale et contrôlée visant à mesurer l'influence du dépistage du cancer de la prostate sur la qualité de vie et la survie, 1002 hommes âgés de 45 à 80 ans ont été choisis au hasard à partir de la liste électorale de la ville de Québec et de sa région [8]. Les hommes ainsi choisis ont été invités par lettre et aucune annonce n'a été faite dans les médias afin d'éviter un biais en faveur d'hommes ayant des symptômes ou une histoire de maladie prostatique.

Un échantillon de sang était obtenu pour la mesure du PSA sérique avant d'effectuer le toucher rectal et l'échographie transrectale de la prostate. Le toucher rectal était fait de façon indépendante du radiologiste qui n'était pas au courant des résultats de PSA sérique ou du toucher rectal. Une biopsie était effectuée lorsque le toucher rectal ou l'échographie transrectale suggérait la présence de cancer. Un PSA sérique au-dessus de $3,0 \mu \mathrm{g} / \mathrm{l}$ identifie une souspopulation d'hommes qui sont à haut risque d'avoir le cancer de la prostate

Un niveau de PSA sérique au-dessus de $3,0 \mu \mathrm{g} / \mathrm{l}$ identifie une souspopulation d'hommes qui sont à haut risque de souffrir du cancer de la prostate. Parmi les 1002 hommes examinés, le cancer de la prostate a été mis en évidence chez 57 d'entre eux, soit une incidence de cancer de la prostate détectable de $5,7 \%$. L'observation probablement la plus importante de cette étude est que 46 de ces 57 cancers $(80,7 \%)$ ont été découverts dans le groupe de 191 hommes ayant un PSA sérique de $3,0 \mu \mathrm{g} / \mathrm{l}$, soit chez seulement $19,1 \%$ de la population totale. De fait, chez le groupe d'hommes ayant un PSA sérique au-dessus de $3,0 \mu \mathrm{g} / \mathrm{l}$, un cancer de la prostate a pu être mis en évidence chez 24,1\% d'entre eux. Par ailleurs, dans le groupe de 811 hommes ayant un PSA sérique égal ou inférieur à $3,0 \mu \mathrm{g} / \mathrm{l}$, seulement $1,4 \%$ d'entre eux ont été découverts comme étant porteurs du cancer de la prostate. En d'autres termes, lorsqu'un homme âgé de 45 à 80 ans a un PSA sérique au-dessus de $3,0 \mu \mathrm{g} / \mathrm{l}$, la probabilité de souffrir d'un cancer de la prostate décelable est de $24 \%$, mais lorsque son PSA sérique est mesuré à $3,0 \mu \mathrm{g} / \mathrm{l}$ ou moins, la probabilité 
d'avoir un cancer de la prostate décelable est 17 fois plus faible, soit chez seulement $1,4 \%$. Ces valeurs sont obtenues avec l'essai d'Hybritech.

Les hommes qui ont un PSA sérique au-dessous de $3,0 \mu \mathrm{g} / \mathrm{l}$ reçoivent la recommandation de revenir de façon annuelle pour mesure de PSA sérique et l'échographie transrectale de la prostate n'est pas effectuée. Bien que le cancer de la prostate eût pu être mis en évidence par échographie transrectale chez $1,4 \%$ des hommes qui ont un niveau de PSA sérique égal ou inférieur à $3,0 \mu \mathrm{g} / \mathrm{l}$, le coût pour mettre en évidence un petit nombre de cancers serait prohibitif car 77 hommes devraient subir cet examen de la prostate afin de découvrir un seul cas de cancer, alors que seulement quatre échographies sont requises pour mettre en évidence un cas de cancer lorsque le PSA sérique est au-dessus de 3,0 $\mu \mathrm{g} / \mathrm{l}$.

Comme le PSA sérique est proportionnel au volume de la tumeur, il est très probable que la plupart des hommes porteurs d'un cancer de la prostate avec un PSA sérique égal ou inférieur à $3,0 \mu \mathrm{g} / \mathrm{l}$ ont un cancer de petit volume encore limité à la prostate qui devrait être encore limité à la prostate et guérissable au moment de sa détection lors d'une prochaine mesure annuelle du PSA sérique. En fait, quand le PSA sérique est à $3,0 \mu \mathrm{g} / \mathrm{l}$ ou moins, la probabilité de ne pas souffrir du cancer de la prostate est de 98,6\%.

Faible coût de détection du cancer de la prostate

En utilisant le PSA sérique comme méthode de prédépistage, le coût pour mettre en évidence un cas de cancer de la prostate est estimé à environ 1500 dollars. Ce coût comprend les mesures du PSA sérique chez 20 hommes $(20 \times 25 \$=$ 500 \$) ainsi que l'échographie transrectale de la prostate chez 4 de ces hommes qui ont un PSA sérique audessus de $3,0 \mu \mathrm{g} / \mathrm{l}(4 \times 250 \$=$ 1000 \$). Ce coût relativement faible de 1500 dollars peut être comparé à celui de 30000 à 50000 dollars requis pour mettre en évidence un cas de cancer du sein par mammographie et de 10000 dollars pour la mise en évidence d'un cas de cancer $\mathrm{m} / \mathrm{s} n^{\circ} 7$, vol. 8 , septembre 92 du col de l'utérus. Lorsqu'il est disponible, le toucher rectal permet d'ajouter un certain nombre de cas de cancer.

L'utilisation du PSA sérique comme méthode de prédépistage permet d'éviter l'ultrasonographie transrectale de la prostate chez $80 \%$ des hommes qui ont une très faible probabilité $(1,4 \%)$ d'avoir un cancer de la prostate décelable tout en réservant cette technique coûteuse à la souspopulation de $20 \%$ des hommes identifiés par un niveau élevé de PSA sérique comme ayant une probabilité beaucoup plus élevée de souffrir du cancer de la prostate $(24,1 \%)$. De plus, il faut noter que l'échographie transrectale de la prostate, bien qu'étant une technique non invasive, a peu de chances d'être acceptable par la population en général comme test annuel de routine pour le dépistage du cancer de la prostate, alors qu'une prise de sang annuelle est facilement acceptable. De plus, la connaissance par un homme d'un niveau de PSA sérique au-dessus de $3,0 \mu \mathrm{g} / 1$ devrait être un stimulus suffisant pour le convaincre de passer à l'étape suivante, soit l'échographie transrectale, afin de confirmer ou d'infirmer la présence de cancer.

Extension possible de 10 années de l'espérance de vie pour chaque homme guéri du cancer de la prostate au stade précoce

Actuellement, dans la majorité des cas $(75 \%)$, le cancer de la prostate est diagnostiqué à un stade avancé après invasion des ganglions lymphatiques et apparition de métastases osseuses (stade D2). L'âge moyen actuel du diagnostic est 65 ans. En utilisant le prédépistage avec le PSA sérique, l'on peut estimer que l'âge du diagnostic pourrait avancer d'au moins 3 années, ce qui amènerait le diagnostic du cancer de la prostate à un âge moyen de 62 ans, la moitié des cancers étant donc découverts entre les âges de 50 et 62 ans. Le diagnostic et le traitement précoces et efficaces du cancer de la prostate pourraient alors potentiellement ajouter, en moyenne, au moins 10 années de vie à chaque homme ainsi traité. Comme un homme sur 11 au cours de sa vie est candidat à cette nouvelle approche, l'espérance de vie moyenne des hommes en général pourrait être ainsi augmentée d'une année. Puisque 132000 nouveaux cas de cancer de la prostate sont découverts annuellement aux États-Unis et que chacun de ces hommes pourrait potentiellement prolonger sa vie de 10 années, plus de 1000000 hommes/année pourrait être ajoutés aux États-Unis, si cette approche s'avérait efficace, alors que le nombre maximal potentiel serait de 100000 par an au Canada et de 30000 par an au Québec. A remarquer que ces chiffres présupposent que tous les hommes soient fidèles au dépistage précoce et que le traitement choisi soit efficace.

Urgence d'évaluer l'efficacité du traitement précoce

Même si le cancer de la prostate est la deuxième cause de décès dus au cancer chez l'homme, très peu d'efforts de recherche ont été consacrés à ce sujet dans le passé. Nous nous retrouvons donc devant la situation où, en 1992, le traitement de ce cancer au stade précoce peut, à la limite, être un choix entre prostatectomie radicale, radiothérapie ou aucun traitement, à cause de l'absence d'études comparatives rigoureuses sur l'efficacité relative des traitements proposés [9]. Il est cependant reconnu que le cancer de la prostate découvert au stade précoce et non traité évolue vers les métastases et le décès dans une proportion importante des cas. En outre, plusieurs données indiquent que, suite à la prostatectomie radicale dans les cas de cancer au stade précoce, l'espérance de vie de ces hommes ainsi traités est comparable à celle des hommes ne souffrant pas du cancer de la prostate [11].

La stratégie de dépistage résumée cidessus offre l'opportunité longtemps attendue d'effectuer des études prospectives et à suffisamment grande échelle pour déterminer avec précision et certitude le meilleur traitement du cancer de la prostate au stade précoce. Tel que clairement énoncé dans un récent éditorial [10], des données fort convaincantes déjà disponibles orientent très fortement vers le traitement précoce du cancer 
de la prostate, une approche qui est d'ailleurs commune à celle déjà reconnue et mise en application dans d'autres types de cancers

Fernand Labrie

Directeur scientifique, Centre hospitalier de l'université Laval, 2705 boulevard Laurier Québec, G1V4G2 Canada.

\section{RÉFÉRENCES}

1. American Cancer Society. Cancer Facts and Figures 1992.

2. Labrie F, Dupont A, Bélanger A. Complete androgen blockade for the treatment of prostate cancer. In : DeVita VT, Hellman S, Rosenberg SA, cds. Important Advan ces in Oncology. Philadelphia : JB Lippincott, 193-217.

3. Crawford D, Eisenberger MA, McLeod $\mathrm{DG}$, et al. A controlled trial of leuprolide with and without flutamide in prostatic carcinoma. N Engl J Med 1989 : 313 : 419-24.

4. Denis L et al. EORTIC 30583 Study. Cancer 1992 (sous presse).

5. Labrie F. Endocrine therapy for prostate cancer. Endocrinol Metab Clin North Am 1991 ; $20: 845-72$.

6. Stamey TA, Kabalin JN, McNeal JE, et al. Prostate specific antigen in the diagnosis and treatment of adenocarcinoma of the prostate. II. Radical prostatectomy treated patients. J Urol 1989 ; 141 : 1076-83.

7. Lee F, Littrup PJ, Torp-Pederson ST, et al. Prostate cancer : comparison of transrectal US and digital rectal examination for screening. Radiology 1988 ; 168 : 389-94.

8. Labrie F, Dupont A, Suburu R, et al. Serum prostate specific antigen as prescreening test for prostate cancer. J Urol 1992 ; $147: 846-52$

9. Labrie F, Lee F, Dupont A. Early stage prostate cancer. Diagnosis and choice of therapy. Excerpta Medica. International Congress Series 841. Amsterdam-New-York : Elsevier ; 1989 : 109 p.

10. Walsh P, Buchanan J. Why make an carly diagnosis of prostate cancer. $J$ Urol 1992 ; $147: 853-4$.

11. Jeweh HJ. Palpable nodule of prostatic cancer. JAMA 1968 ; 203 : 403-6. 